\title{
Dynamical Cognitive Modeling of Syntactic Processing and Eye Movement Control in Reading
}

\author{
Maximilian M. Rabe ${ }^{1}$, Dario Paape ${ }^{2}$, Shravan Vasishth ${ }^{2,3}$, and Ralf Engbert ${ }^{1,3}$ \\ ${ }^{1}$ Department of Psychology, University of Potsdam \\ ${ }^{2}$ Department of Linguistics, University of Potsdam \\ ${ }^{3}$ Research Focus Cognitive Sciences, University of Potsdam
}

\begin{abstract}
Integrating eye-movement control and sentence processing would mark an important step forward for mathematical models of natural language processing. We present an integrated approach by combining the SWIFT model of eye-movement control (Engbert et al., 2005) with key components of the LV05 (Lewis \& Vasishth, 2005) parser. The integrated generative model can reproduce reading time patterns that have been explained in terms of similarity-based interference in the psycholinguistic literature. A crucial problem for such complex models is parameter estimation. We build upon recent advances on successful parameter identification in dynamical models, investigate likelihood profiles for single parameters, and present pilot results on MCMC sampling within a Bayesian framework of parameter inference.
\end{abstract}

Keywords: Reading, eye-movement control, sentence processing, dynamical models, Bayesian inference, oculomotor control, individual differences

When reading text, words are fixated for approximately 150 to $300 \mathrm{~ms}$ on average, and thus about 3 to 4 saccades are generated per second (Rayner, 1998). Readers tend to make about as many fixations as the sentence has words, but fixation sequences are not always linear: Instead of systematically shifting the gaze from one word to another, which only happens in about $50 \%$ of fixations, readers also skip words, refixate the same word, or regress back to previously fixated regions of text. Reading models should be able to account for, explain, and generate such patterns.

There is considerable evidence that syntactic processing influences eye guidance during reading (e.g., Clifton et al., 2007; Frazier and Rayner, 1982). Although several mathematical models of eye-movement control exist, they generally ignore the costs arising due to parsing difficulty. Previous attempts at integrating models of parsing difficulty with eye-movement control include

Correspondence should be addressed to Maximilian M. Rabe (D) https://orcid.org/0000-0002-2556-5644, maximilian.rabe@uni-potsdam.de, University of Potsdam, Department of Psychology, Karl-Liebknecht-Str. 24/25, 14476 Potsdam, Germany. 
variants of the E-Z Reader model (Reichle et al., 1998), e.g., in combination with a retrieval- based parser (Engelmann et al., 2013; Vasishth \& Engelmann, 2021), and Über-Reader (Veldre et al., 2020). However, rigorous statistical inference on parameters for models of such complexity is an additional research topic (Schütt et al., 2017). Below, we present an integration of the SWIFT model of eye-movement control (Engbert et al., 2005) with the LV05 model of sentence processing (Lewis $\&$ Vasishth, 2005), and show that robust parameter recovery is possible for the combined model.

\section{The SWIFT model of eye-movement control}

A major characteristic of SWIFT is the parallel processing of words (Engbert et al., 2002). Generally, the model assumes that all words that fall within a processing span around the current fixation location are processed in parallel but the processing rate $\Lambda_{n}(t)$ of any given word $n$ at time $t$ depends on a number of factors such as gaze eccentricity. Each word in the sentence passes through a lexical and post-lexical processing stage. During lexical processing, word recognition and identification take place. As word recognition is ongoing, the activation $a_{n}(t)$ associated with the processed word $n$ rises up to a maximum threshold modulated by the word's corpus frequency. Once the word is identified, post-lexical processing begins and word activation decreases. ${ }^{1}$ During this stage, higher-level cognitive processing is assumed to take place.

Saccade target selection in SWIFT is inherently stochastic, as it depends on the dynamic, relative word activations at any given point in time. Words with high activation values are more likely to be selected as targets than words with lower activation. The probability $\pi_{n}(t)$ to select word $n$ at time $t$ as the next saccade target is given as

$$
\pi_{n}(t)=\frac{a_{n}(t)}{\sum_{m} a_{m}(t)}
$$

where $a_{n}(t)$ is the activation of word $n$ at time $t$ and its differential over time is the processing rate $\Lambda_{n}(t)$.

The relation between the activation $a_{n}(t)$ of a word and its selection probability $\pi_{n}(t)$ also entails that words requiring little processing (i.e., easy words) pass through lexical and post-lexical processing faster than less frequent (i.e., difficult) words, are in a state of higher activation for a shorter time period, are consequently less likely to be fixated, and thus often skipped, for example.

While saccade target selection mainly depends on word activations, the timing of saccades is relatively independent process (Findlay \& Walker, 1999). The cascade starts with a global timer, which triggers the labile and subsequent non-labile saccade stages-a distinction motivated by oculomotor performance in the double-step paradigm (Becker \& Jürgens, 1979). Different circumstances can lead to cancelation of saccades and continuous target selection changes. At the end of the labile saccade stage, a cancellation of the saccade is no longer possible and the target is invariable. However, the actual saccade execution is delayed until the end of the non-labile saccade stage. The subsequent execution of the saccade itself is a noisy process subject to systematic and random error (McConkie et al., 1988), which is compatible with Bayesian model of oculomotor control (Engbert \& Krügel, 2010).

\footnotetext{
${ }^{1}$ It is possible to include more detailed processes of word recognition in models of eye-movement control, e.g., the open bigram model in OB1-Reader (Snell et al., 2018), if letter-level effects seem relevant to a specific problem in eye-movement control.
} 
In a recent article (Rabe et al., in press), we developed a fully Bayesian framework for parameter inference of the SWIFT model of eye-movement control. Results were based methodologically on the development of a likelihood approximation (Seelig et al., 2020). Using large-scale numerical simulations, it was shown that the model could reliably reproduce fixation durations, fixation probabilities and saccade amplitudes at the level of global and by-subject summary statistics, even without an explicit mechanism for any higher-level cognitive processes such as syntactic parsing. Given these promising results, the present work uses SWIFT as a fundamental model component to account for lower-level characteristics of fixation sequences that may otherwise obfuscate the more subtle observable effects of higher-level cognition.

\section{Sentence processing and cue-based memory retrieval}

During sentence reading, the human sentence parsing system has to incrementally integrate individual words into a syntactic structure, and sentence meaning has to be derived compositionally. One reason why disruption of the reading process, as indexed by increased fixation durations and regressions, occurs is when the parser has difficulty integrating incoming words with previous input. Prominent examples of such phenomena in reading include garden-path effects (e.g., Frazier and Rayner, 1982), local coherence effects (e.g, Tabor et al., 2004), and similarity-based interference effects (e.g., Gordon et al., 2006; Van Dyke and McElree, 2006).

Lewis and Vasishth (LV05; 2005) proposed a model of sentence processing that is based on the ACT-R architecture (Anderson \& Lebiere, 1998; Anderson, 2005). The LV05 model assumes that the post-lexical integration of words and working memory are interactively linked. Words are integrated into syntactic constituents that are stored in memory as chunks. Sentences often exhibit non-local dependencies between incoming words and stored constituents, such as in (1), where the noun phrase the robber has to be connected to the verbs (was) chasing and escaped.

(1) The robber that the policeman in the patrol car was chasing escaped.

In the LV05 model, encountering a new word that has some syntactic dependency, such as escaped in the above example, triggers a search in memory for a suitable dependent. The search process is cue-based, that is, the verb specifies a set of linguistic features such as \pm noun or \pm animate to identify the correct dependent, and existing memory chunks are reactivated based on their feature specifications. The best-matching candidate is usually retrieved, but when multiple memory chunks match the retrieval cues and compete for activation, such as the robber and the policeman in (1), processing is slowed due to the so-called fan effect (e.g., Anderson, 1990).

The LV05 model has been used to model a variety of phenomena in the sentence-processing literature (see Engelmann et al., 2019 for a review). However, as LV05 does not have a module for oculomotor control, reading is modeled in a purely incremental, left-to-right fashion. Attempts at integrating LV05 with explicit models of eye-movement control have been made by Engelmann et al. (2013) and Vasishth and Engelmann (2021), who combined LV05 with EMMA (Salvucci, 2001), and Dotlačil (2018), whose model is based purely on ACT-R. These models capture many reading patterns in real-world data but nevertheless leave room for improvement.

\section{Integration of SWIFT and LV05}

The processing difficulty of words in SWIFT primarily depends on lower-level word characteristics such as corpus frequency and word length (Kliegl et al., 2004). While SWIFT keeps 
track of the processing stage of words in the sentence, it has no higher-level mental representation of the constituents or of the entire word sequence. Adjacent words may have an influence on processing difficulty, but there is no mechanism which would predict or account for difficulties due to dependency completion processes on the sentence level.

The integration of a memory retrieval process as a systematic link between word identification and saccade targeting is a promising starting point for improving the goodness-of-fit of the SWIFT model and providing insights into empirical phenomena from the sentence-processing literature (Engelmann et al., 2013). For the integration of the interaction, we opted for a simplified version of the LV05 model (Engelmann, 2015) and the most current baseline version of SWIFT (Rabe et al., in press). The resulting SWIFT-LV05 model uses the baseline eye-movement control architecture of SWIFT and the interactive working memory module of LV05. Both models interact so that SWIFT determines the memory schedule for LV05 and chunk activations computed by LV05 modulate word activations in SWIFT.

It should be noted that both models use the term activation in slightly different ways. Activations in SWIFT and LV05 have in common that high values determine the most likely candidate for some selection process. We will refer to SWIFT activations as lexical activations and to LV05 activations as memory activations. In SWIFT, every word is associated with a lexical activation value at any given point in time. Word activations rise during the lexical processing stage and decrease during the post-lexical processing stage. Once they return to zero, words are considered to be fully processed. The relative activations at any point in time determine the probability for each word to be selected as the next saccade target. Due to the underlying activation dynamics, words are most likely to be selected as saccade targets when they are transitioning between lexical and post-lexical processing but they may also be selected otherwise, especially if the other words in the sentence have lower activations at the time of saccade programming. Additionally, misplaced fixations are generated frequently due to saccadic errors, so that 10 to $15 \%$ of the fixations occur on unintended words (Engbert \& Nuthmann, 2008).

In LV05, memory chunks have activation values that are determined by recency and by feature match during retrieval events. Memory chunks are created as words in the sentence are processed, but memory can also hold encodings that do not originate from the sentence (see below). During retrieval, the memory item with the highest memory activation is matched for the dependency to be resolved. The latency of the retrieval is inversely proportional to the memory activation of the retrieval target. Memory activation spikes when chunks respond to retrieval probes via cue matching and decays exponentially over time.

In the integrated model, the timeline of a word starts with the lexical processing stage. Once lexical processing finishes, the word is encoded in working memory along with its linguistic features (e.g, \pm noun, \pm animate) and transitions into the post-lexical stage. Words with syntactic dependencies trigger a retrieval process after SWIFT's post-lexical processing stage. Linguistic dependencies are resolved sequentially, and processing is complete when all dependencies have been resolved.

\section{Mathematical formulation of the integrated model}

The integrated SWIFT-LV05 model is based on the most recent SWIFT implementation (Engbert et al., 2005; Rabe et al., in press; Seelig et al., 2020) and a simplified version of the LV05 model (Engelmann, 2015; Lewis \& Vasishth, 2005). The model assumes an overall effect of memory load on the processing rates of all words. Generally, memory load is high when there are 
many active, non-decayed items. Items become active when words are encoded or when retrievals are performed, and remain active when decay is slow. The overall memory load $B(t)$ is a function of time so that

$$
B(t)=\sum_{i} \exp \left(-d \cdot\left\lfloor t-t_{i}\right\rfloor\right),
$$

where $d>0$ is the decay parameter, $t_{i}$ is the time of the $i$-th memory access, and

$$
\left\lfloor t-t_{i}\right\rfloor:=\left\{\begin{array}{cl}
t-t_{i} & \text { if } t \geq t_{i} \\
\infty & \text { if } t<t_{i}
\end{array}\right.
$$

so that the contribution of the $i$-th memory access to the activation, $\exp \left(-d \cdot\left\lfloor t-t_{i}\right\rfloor\right)=0$ for $t<t_{i}$, i.e., memory access has not yet occurred. The modulated processing rate $\Lambda_{n}^{\star}$ for word $n$ is given by

$$
\Lambda_{n}^{\star}(t)=\Lambda_{n}(t) \cdot \exp \left(-\mu_{1} B(t)\right) .
$$

When a dependency needs to be resolved, a retrieval is triggered after post-lexical processing finishes. At that time, the lexical activation of the retrieval trigger is reset to some fraction of its maximum value. The processing rate for the retrieval stages is additionally modulated by the LV05 memory activation so that

$$
\Lambda_{n}^{\star \star}(t)=\Lambda_{n}^{\star}(t) \cdot \exp \left(\mu_{2} R_{n}(t)\right)=\Lambda_{n}(t) \cdot \exp \left(\mu_{2} R_{n}(t)-\mu_{1} B(t)\right),
$$

where $R_{n}(t)$ is the memory activation for the retrieval trigger $n$ at time $t$. Activation $R_{n}(t)$ is equal to the memory activation $A_{k}(t)$ of retrieval candidate $k$ at time $t$. Item index $k$ changes dynamically over the course of the retrieval by the relative memory activations $A_{k}(t)$ of all retrieval candidates. Therefore, the candidate with the highest activation is most likely to determine the retrieval activation $R$ at time $t$. Once the lexical activation of memory item $k$ has returned to zero and the retrieval is complete, item $k$ is determined as the retrieved memory item. This is consistent with the LV05 model, since the memory item with the highest activation is the most likely to be matched for the dependency and determine the retrieval latency.

The memory activation $A_{k}(t)$ of memory item $k$ corresponds to the retrieval activation in the LV05 model so that

$$
A_{k}(t)=S_{k}(t)+P_{k}(t)+B_{k}(t),
$$

where $S_{k}$ is the memory association strength, $P_{k}$ is the mismatch penalty, and $B_{k}$ is the item-specific baseline activation. The memory association strength is computed as the sum over the fan effects $\phi_{k . l}(t)$ of all $l$ features of memory item $k$ so that

$$
S_{k}(t)=\sum_{l}\left[S_{\max }-\log \phi_{k . l}(t)\right],
$$

where $S_{\max }$ is the maximum activation strength parameter of the LV05 model and $\phi_{k . l}(t)$ is defined as the number of memory items with feature $l$ at time $t$, including memory item $k$ itself so that $\phi_{k . l}(t) \geq 1$.

The mismatch penalty adds an additional penalty for all retrieval cues $l$ that do not match the corresponding feature of memory item $k$, i.e.,

$$
P_{k}(t)=\sum_{l} \Delta_{k l}
$$




\section{Figure 1}

Example illustration of the baseline activation (memory load) as a function of time.

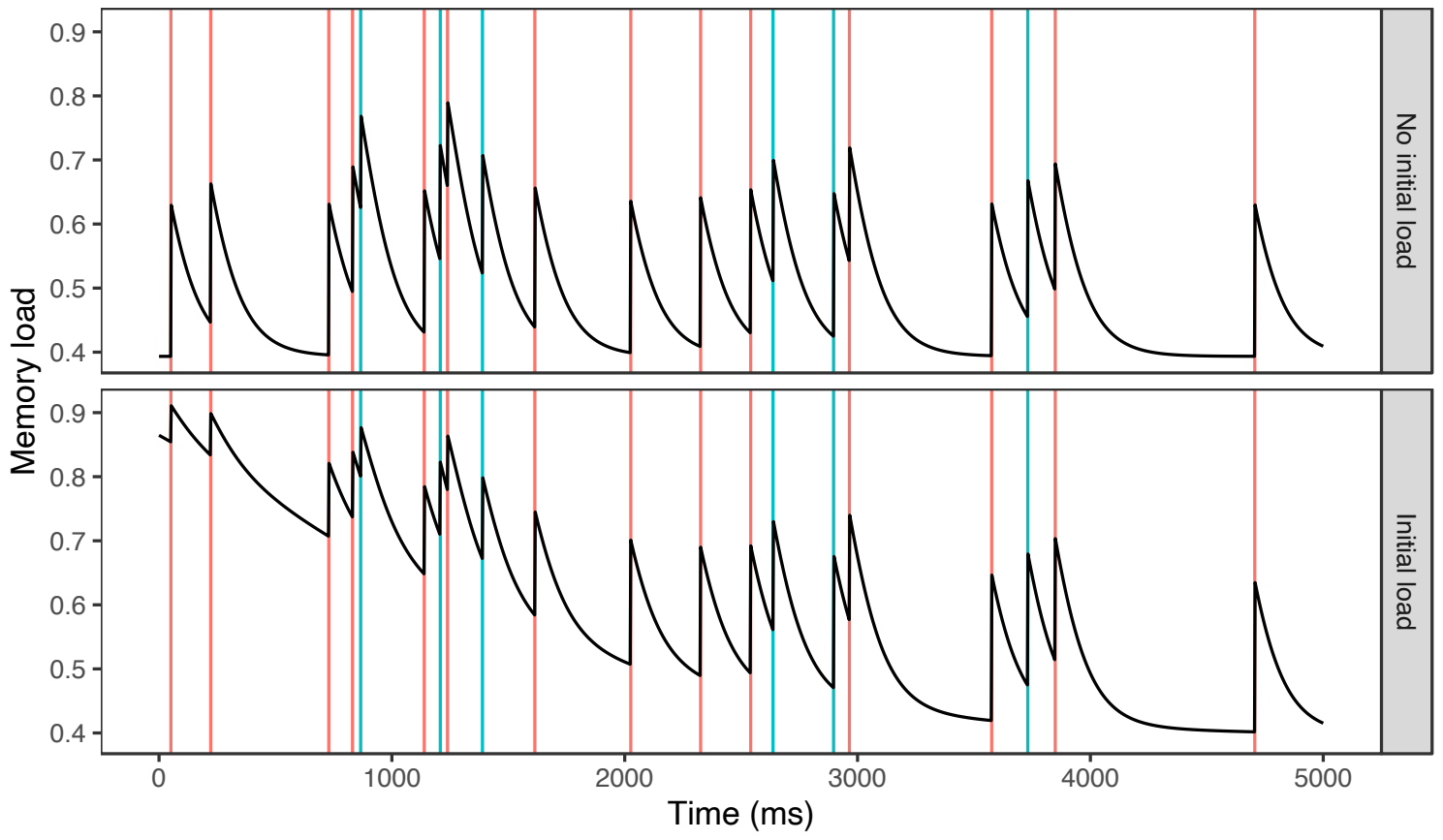

Note. Memory load as the inverse baseline activation $B(t)$. Vertical lines are memory access times (encodings in red and retrievals in blue). The top panel shows a trial without initial memory load, the bottom panel shows a trial words memorized before sentence reading.

where

$$
\Delta_{k l}:=\left\{\begin{array}{ccc}
0 & \text { if } & \text { cue }_{l}=\text { feature }_{k l} \\
-p & \text { otherwise }
\end{array}\right.
$$

and $p>0$ is a free parameter specifying the single mismatch penalty.

The calculation of the baseline activation of a specific memory item $k$ parallels the calculation of the overall memory load $B(t)$ and is

$$
B_{k}(t)=\sum_{i} \exp \left(-d \cdot\left\lfloor t-t_{i k}\right\rfloor\right),
$$

where $t_{i k}$ is the $i$-th memory access (encoding or retrieval) of memory item $k$. Therefore, the overall memory load $B(t)$ is equal to the sum of all $B_{k}(t)$ if all memory access times are matched to a memory item. Figure 1 illustrates the time course of the baseline activation (memory load) in a simulated trial with or without initial memory load. Events of memory access induce memory load, which in turn decays exponentially over time. Even though the two trials start out at different initial levels of memory load, the memory load condition can be seen to approach the no-load condition after some time, which is when the initial memory load has mostly decayed.

If a word triggers more than one retrieval (such as a verb that needs both a subject and an object), the retrievals occur sequentially; after the first retrieval has completed, the second is conducted, and so on. If there are no memory items that could possibly match the retrieval, for 
example when none have been encoded yet, the retrieval pauses at its initial lexical activation value until a retrieval candidate enters memory. An example model simulation can be seen in Figure 2.

\section{Predictions of the integrated model}

The integrated model makes specific qualitative and quantitative predictions for a number of psycholinguistic phenomena, namely memory load and memory interference. Given that the processing rate is modulated by the overall memory load (or recency of past memory access times), the memory-dependent processing rate $\Lambda_{n}^{\star}(t)$ will be lower than the baseline processing rate $\Lambda_{n}(t)$, if items have recently been encoded or accessed in memory, as there are necessarily more memory access times. This can, for example, be tested by increasing the memory load with encodings before reading starts and instructing the reader to retain those encodings for the duration of the trial. In such a setting, memory load will be higher relative to an identical sentence without prior encodings, and thus processing should be slower and reading times should be longer, especially at the beginning of the trial.

The free parameters $\mu_{1}$ for processing modulation and decay $d$ determine the strength of the overall memory load effect. As one may assume that some memory items decay faster or more slowly than others, e.g., due to different task demands, it is useful to consider different values for $d$.

Memory interference occurs when multiple items in memory compete for the same retrieval cues, as the fan effect increases. This may be caused by previously processed words within the sentence or memory items encoded before reading. The resulting $R_{n}(t)$ and thus $\Lambda_{n}^{\star \star}(t)$ will on average be lower compared to a sentence with reduced or non-interfering memory load. The interference effect cannot occur without interfering memory contents and will especially be modulated by parameter $\mu_{2}$.

\section{Methods}

\section{Experimental data}

To test the integrated model, its predictions, and explanatory power with regard to psycholinguistic data, we use empirical data from an experimental study of proactive memory interference during sentence processing. In a large-scale attempt to replicate earlier findings by Van Dyke and McElree (2006), Mertzen et al. (2020) displayed sentences to subjects in four different experimental conditions in a crossed memory load $(2) \times$ feature match $(2)$ design. Sentences were presented either with memory load $(2 \mathrm{a}, \mathrm{b})$ or without memory load $(2 \mathrm{c}, \mathrm{d})$. In the memory load

conditions, participants were instructed to memorize a set of three nouns before sentence reading began. The three memory items were designed for each sentence so that they either were plausible syntactic objects for the critical verb sailed/fixed in (2b) or not (2a).

(2) a. [TABLE SINK TRUCK] It was the boat that the guy who lived by the sea sailed in two sunny days.

b. [TABLE SINK TRUCK] It was the boat that the guy who lived by the sea fixed in two sunny days.

c. It was the boat that the guy who lived by the sea sailed in two sunny days.

d. It was the boat that the guy who lived by the sea fixed in two sunny days. 
Figure 2

Segment of internal word-based activation profiles for an example simulation run with the integrated SWIFT-LV05 model

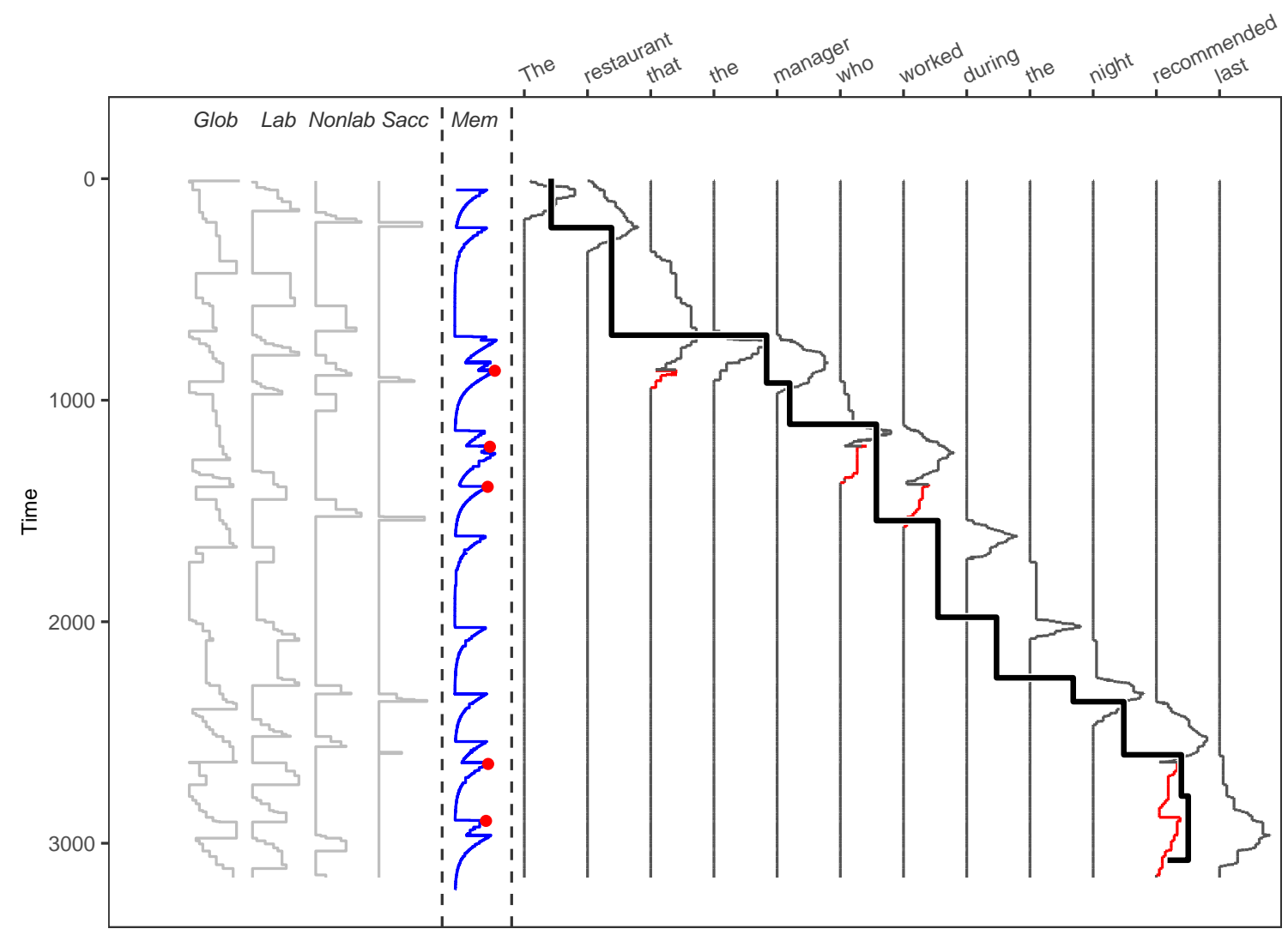

Note. Time is increasing on the vertical axis from top to bottom. Vertical lines indicate oculomotor processes (light grey, left), memory load (blue), and word activations (dark grey, right). The eye's trajectory (black line, bold) is driven by activation-based, stochastic target selection. Plotted within-word fixation positions are normalized by word length. Memory load $B(t)$ increases with each memory access and decays over time with rate $d$. Retrieval processes are highlighted via red dots along the trace of the memory load $B(t)$ and produce increasing word-activations of the word triggering a retrieval. In the example, the two retrievals triggered by word 11 at about $2,500 \mathrm{~ms}$ produce several refixations on the retrieval trigger, which are a result of stochastic target selection of the SWIFT model. 


\section{Figure 3}

Predictions for mean first-pass reading times (FPRT, gaze durations) for four different experimental conditions.
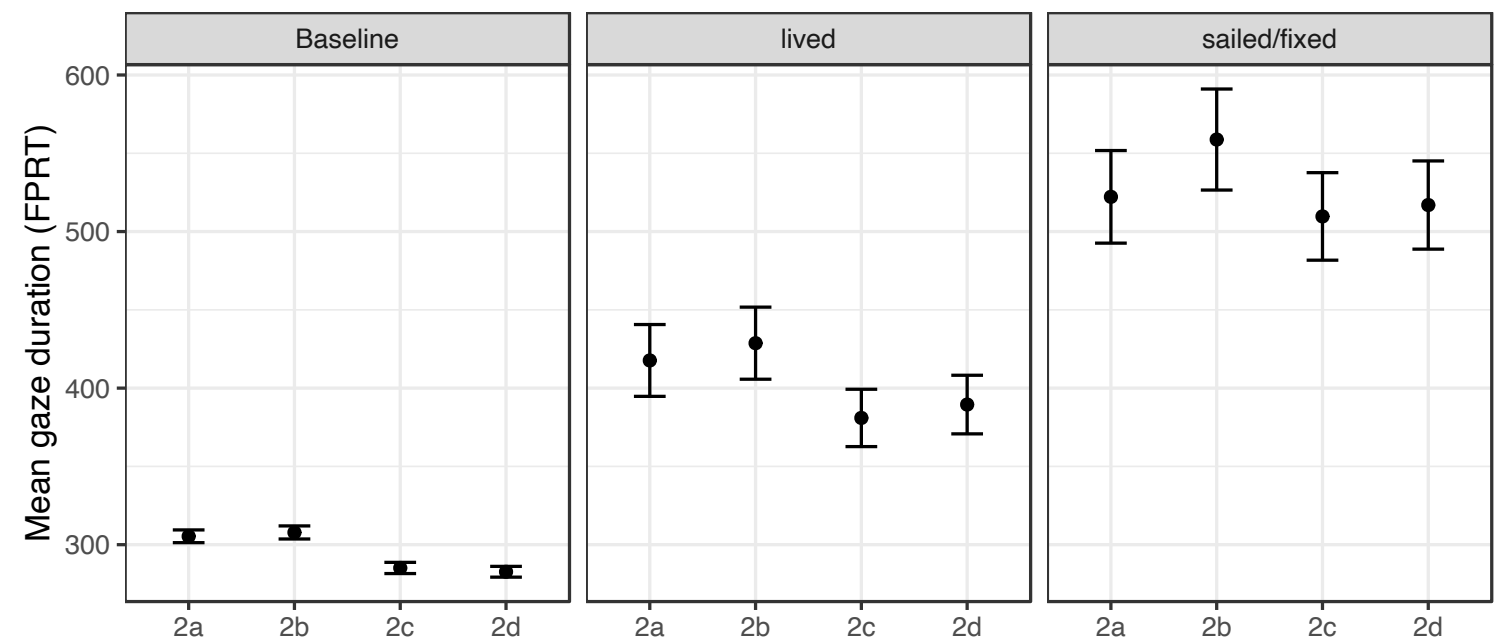

Note. Results are grouped by fixated region (see 2), where the baseline summarizes all words outside the regions of lived and fixed/sailed. Conditions (2a,b) have memory load, $(2 \mathrm{c}, \mathrm{d})$ do not. $(2 \mathrm{~b}, \mathrm{~d})$ are interference items, $(2 \mathrm{a}, \mathrm{c})$ are not.

At the critical verb fixed in condition (2b), the memory items should compete with the sentence-internal noun (boat) as plausible objects of the verb, causing proactive memory interference and creating processing difficulty. By contrast, at the verb sailed in condition (2a), it should be easier to retrieve boat because the memory items do not match the semantic features of the verb. Conditions $(2 \mathrm{c}, \mathrm{d})$ serve as control conditions for the memory interference effect, and also to investigate effects of memory load irrespective of feature match between verbs and their potential objects.

As a proof of concept that the integrated SWIFT-LV05 is generally capable of generating the predicted pattern of effects, data were simulated for the sentences used in Mertzen et al. (2020). Summary results are illustrated in Figure 3, which plots first-pass reading times (FPRT) for words across four different experimental conditions. Words are grouped by theoretical number of triggered retrievals. In conditions ( $2 a)$ and $(2 b)$, the model memorized three words before reading started. In conditions $(2 \mathrm{c})$ and $(2 \mathrm{~d})$, working memory was empty at the beginning of the trial. Sentences were identical in $(2 \mathrm{a}, \mathrm{c})$ as well as in $(2 \mathrm{~b}, \mathrm{~d})$.

Regarding the summary statistics of the simulated data, there is a memory load effect for all words such that mean gaze durations are higher for conditions $(2 a, b)$ vs. $(2 c, d)$. The interference effect is observable for the critical verb causing two retrievals but only in sentences where the model memorized interfering items before reading (2b). This demonstrates that the SWIFT-LV05 is generally capable of generating memory interference and load effects. 


\section{Computer implementation}

We translated the simplified LV05 algorithm in R (Engelmann, 2015) to C for performance and compatibility with the most recent SWIFT implementation (Rabe et al., in press), which is also implemented in the $\mathrm{C}$ programming language. This computationally efficient framework permits high-performance computing such as for MCMC sampling. The resulting memory retrieval module based on LV05 can be switched on and off in the final model so that an easy comparison between the baseline SWIFT model and the integrative SWIFT-LV05 model is possible.

\section{Bayesian parameter inference}

The reliability of computational cognitive models depends critically on the availability of adequate methods for statistical inference (Engbert, 2021; Schütt et al., 2017). Experimental data in eye-movement research are fixation sequences consisting of time-ordered observations. In this case, the identification of model parameters is done within the field of data assimilation (Reich \& Cotter, 2015). Within this framework, the SWIFT model has been implemented for Bayesian parameter inference (Seelig et al., 2020). Subsequently, it could be demonstrated in a principled Bayesian workflow that the model can be reliably fitted to simulated and experimental data even with many free parameters and sparse data due to splitting by experimental condition (Rabe et al., in press). As the LV05 mechanisms have been fully integrated into the SWIFT architecture, we can generally use the same procedures for parameter inference for the integrated SWIFT-LV05 model.

In the present study, experimental conditions on memory load and feature match are directly implemented as model processes. We are therefore not considering statistical effects in model parameters between experimental conditions but are instead fitting the additional parameters that specify the relation between the SWIFT and LV05 modules. Using this approach, the approach gains explanatory and statistical power, as a single model is fitted to all experimental conditions at once rather than separately by conditions.

Words that enter memory due to memorizing may be assumed to decay in memory at a different rate than words processed during sentence reading. For this purpose we are not fitting a single parameter value for $d$ but parameter $d_{1}$ for memory items encoded after reading began and parameter $d_{2}$ for words encoded before reading the sentence.

As the integration of the SWIFT-LV05 model effectively modifies model dynamics and thus the likelihood function, a reevaluation of the likelihood profiles is critical. Consequently, the new likelihood function may be used for Bayesian parameter inference. A parameter recovery using the targeted sampling algorithm DREAM ZS (Laloy \& Vrugt, 2012; ter Braak \& Vrugt, 2008; Vrugt et al., 2009) using simulated data with known true parameter values is performed to ensure the validity of the computational approach. After successful evaluation of likelihood profiles and parameter recovery, the model may be fitted to empirical data.

\section{Results}

\section{Likelihood profiles}

In order to assess whether the described model modification is appropriately captured in its likelihood, it should be ensured that the newly introduced free parameters of the integrated LV05 model component affect the outcome likelihood. Thus, the behavior of the likelihood as a func- 


\section{Figure 4}

Stochastic log-likelihood profiles for different free parameters and a single simulated data set with known (true) parameters

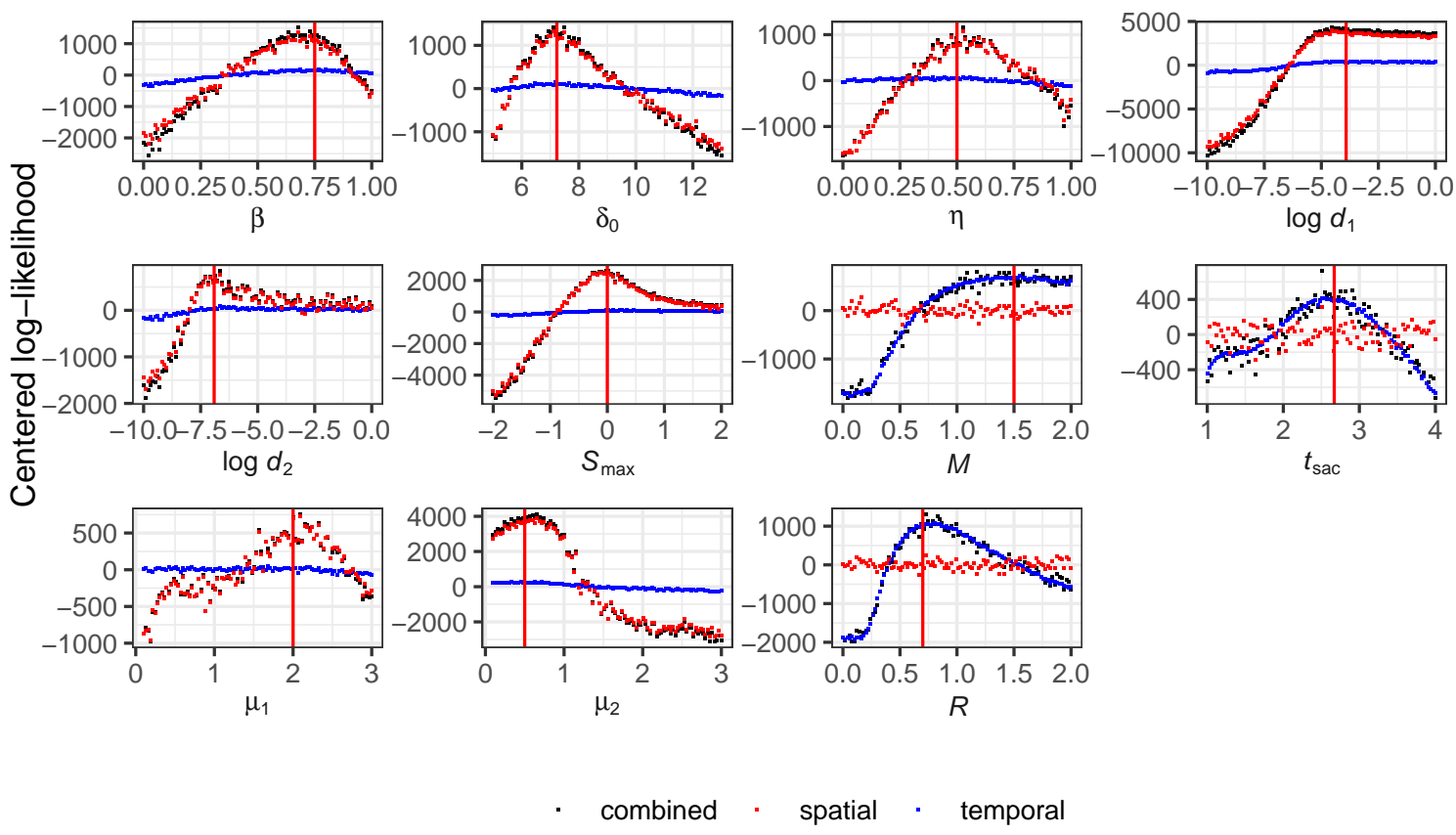

Note. Log-likelihood components are centered around their respective mean and are evaluated along the free parameter on the respective horizontal axis by keeping all other parameters constant at the value indicated by their respective true value (red vertical line). Colors of dots represent the result for the specific log-likelihood component (spatial in red, temporal in blue) or their combined sum (black).

tion of each of the new parameter represent a necessary condition for identifiability and statistical inference of the full model (Rabe et al., in press; Seelig et al., 2020).

Generally, the likelihood $L_{M}(\theta \mid X)$ for parameters $\theta$ given data $X$ should be maximal for the known true parameters with which $X$ was generated. Therefore, when we simulate a dataset $X$ with known parameters $\theta_{\text {true }}$ and then evaluate $L_{M}(\theta \mid X)$ along some continuum $\theta$ including the true value, we should observe that $L_{M}(\theta \mid X)$ peaks at $\theta=\theta_{\text {true }}$.

In Figure 4, it can be seen that the likelihood for the integrated model does indeed peak at the known true parameter values (vertical red lines), while holding all other parameters constant. Moreover, it can be observed that the new parameters $d_{1}, d_{2}, S_{\max }, \mu_{1}$, and $\mu_{2}$ predominantly affect the spatial likelihood component with small effects on the temporal likelihood.

\section{Parameter recovery}

The successful likelihood profile evaluation is a critical first step toward the assessment of the validity of the parameter inference procedure. For a complete assessment, however, especially when fitting many parameters at once, we must evaluate the computational faithfulness of the entire fitting procedure. In addition to the evaluation of the mere likelihood profiles, we are therefore simulating many datasets with true parameters randomly sampled from a prior distribution. Recovering 


\section{Figure 5}

True and recovered parameters for $N=31$ simulated datasets. Vertical bars are $60 \%$ HPDIs and dots are their respective midpoints. The diagonal line refers to identity.
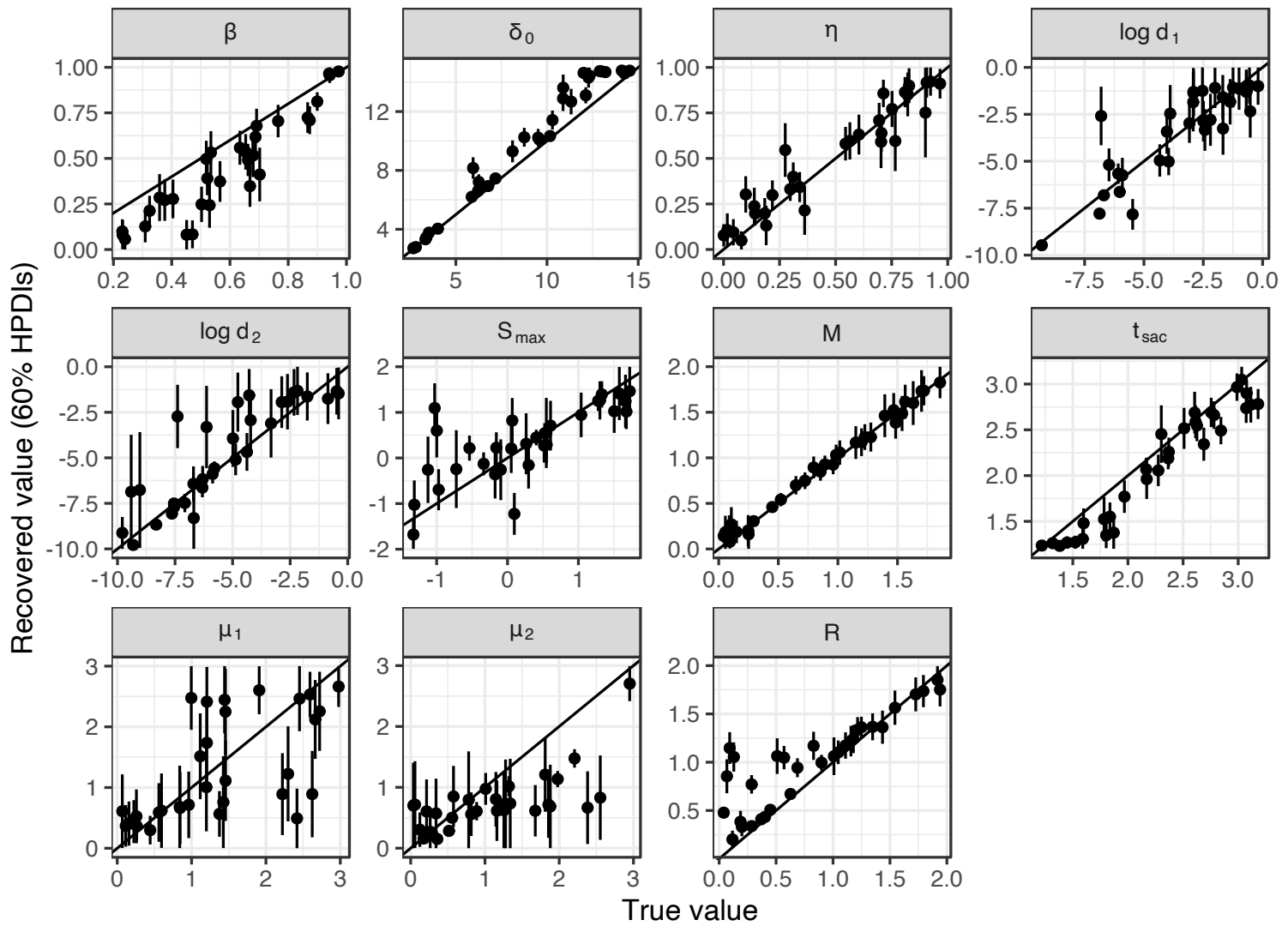

parameters from these simulated datasets by fitting the model in the same framework to be used later, ensures that the method is generally valid.

The parameter recovery study shows relatively promising outcomes, as presented in Figure 5. Most importantly, the baseline SWIFT parameters, as previously reported in Rabe et al. (in press), are reliably recovered as the true values are mostly within the highest posterior density intervals (HPDIs) of the recovered values. Among the new parameters, the memory decay parameters $d_{1}$ and $d_{2}$, as well as $S_{\max }$ are also reliably recovered. The exponential modulator parameters $\mu_{1}$ and $\mu_{2}$ are more problematic. Their computational faithfulness may need improvement should they need to be fitted.

\section{Discussion}

The goal of the current article was to propose the foundations of an integrated model of eyemovement control and syntactic processing and to analyze its statistical properties and predictions. Reading models often do not explicitly address interaction of oculomotor control and higher-level sentence processing. A major challenge for building integrative models is the added complexity and difficulty in optimizing many model parameters simultaneously in a dynamical model (Engbert, 
2021). Bayesian parameter inference is useful in solving these problems as it naturally restricts the space of plausible parameters via prior distributions (Rabe et al., in press; Schütt et al., 2017).

Recently, the SWIFT model of eye-movement control during reading (Engbert et al., 2005) has been implemented for Bayesian parameter inference (Seelig et al., 2020) and shown to produce reliable parameter estimates and convincing fits of relevant summary statistics within a principled Bayesian workflow (Rabe et al., in press). Therefore, SWIFT is a useful candidate for the integration of further higher-level sentence processing, such as the LV05 parsing model (Lewis \& Vasishth, 2005). By using a simplified implementation of LV05, we demonstrated that such an integration is practically feasible, produces relevant outcomes for the investigation of experimentally observed psycholinguistic phenomena, and provides an objective, process-oriented explanation of the findings.

We introduced an activation-based coupling of the two model components. While internal activation values have specific interpretations in each of the models, it is possible to formulate an interaction, where memory activations in LV05 modulate word activations in the SWIFT model. In the integrated model, activations in the parser reflect the construction of a sentence representation, which influences word activations and saccade-targeting in the eye-movement model. Therefore, rather than assuming that the parser has direct control of the eye-movement targeting system, we propose an indirect, stochastic influence on saccade goals via memory activations. This is in line with our results on rather moderate effects of syntactic processing on eye guidance (Mertzen et al., 2020), and contrasts with earlier claims of a more direct relationship (Frazier \& Rayner, 1982; Just \& Carpenter, 1980).

More specifically, we showed that the integration of the simplified LV05 module allows the SWIFT architecture to handle higher-level processing. We simulated fixation sequences with the integrated computational model and investigated whether parameters could be recovered from the simulated data. We found that newly introduced model parameters that are based on the original LV05 parameters are identifiable via likelihood profiles and can be reliably recovered using MCMC sampling.

The finding that parameter values can be reliably recovered for the new LV05 parameters is particularly remarkable as they only influence the processing rate of words, i.e., the temporal gradient of their SWIFT activations. Even so, they affect both temporal and spatial summary statistics and are able to predict phenomena of interest such as memory interference effects. These preliminary results motivate further development of the likelihood function, more robust specification of model parameters, and fitting the model to additional experimental data in order to describe and explain empirical effects.

\section{Conclusion}

Syntactic processing and eye-movement control must be coordinated during reading to serve language processing. We developed an integrated computational model that bridges the gap between the two fields of research. The integrated model makes specific predictions for effects of memory and retrieval processes on eye movements during reading. Based on simulated fixation sequences, model parameters can be reliably recovered. Our approach provides rigorous statistical methods for parameter inference from experimental data. 


\section{Acknowledgments}

This work was supported by a grant from Deutsche Forschungsgemeinschaft to Ralf Engbert and Shravan Vasishth (SFB 1297 Variability in Language, project no. 317633480). We also acknowledge support by Norddeutscher Verbund für Hoch- und Höchstleistungsrechnen (HLRN, project no. bbx00001) for providing high-performance computing resources.

\section{References}

Anderson, J. R., \& Lebiere, C. (1998). Atomic components of thought. Lawrence Erlbaum Associates.

Anderson, J. R. (2005). Human symbol manipulation within an integrated cognitive architecture. Cognitive Science, 29(3), 313-341. https://doi.org/10.1207/s15516709cog0000_22

Anderson, J. R. (1990). Cognitive psychology and its implications. Freeman.

Becker, W., \& Jürgens, R. (1979). An analysis of the saccadic system by means of double step stimuli. Vision Research, 19(9), 967-983. https://doi.org/10.1016/0042-6989(79)90222-0

Clifton, C., Staub, A., \& Rayner, K. (2007). Eye Movements in Reading Words and Sentences. In R. V. Gompel, M. Fisher, W. Murray, \& R. L. Hill (Eds.), Eye movements: A window on mind and brain. Elsevier.

Dotlačil, J. (2018). Building an act-r reader for eye-tracking corpus data. Topics in Cognitive Science, 10(1), 144-160.

Engbert, R. (2021). Dynamical models in neurocognitive psychology. Springer Nature International Publishing. https://www.springer.com/de/book/9783030672980

Engbert, R., \& Krügel, A. (2010). Readers use Bayesian estimation for eye movement control. Psychological Science, 21(3), 366-371. https://doi.org/10.1177/0956797610362060

Engbert, R., Longtin, A., \& Kliegl, R. (2002). A dynamical model of saccade generation in reading based on spatially distributed lexical processing. Vision Research, 42(5), 621-636. https: //doi.org/10.1016/S0042-6989(01)00301-7

Engbert, R., \& Nuthmann, A. (2008). Self-consistent estimation of mislocated fixations during reading. PLoS One, 3(2), e1534. https://doi.org/10.1371/journal.pone.0001534

Engbert, R., Nuthmann, A., Richter, E. M., \& Kliegl, R. (2005). SWIFT: A dynamical model of saccade generation during reading. Psychological Review, 112(4), 777-813. https://doi.org/ 10.1037/0033-295x.112.4.777

Engelmann, F. (2015). ACTR-in-R [GitHub repository]. https://github.com/felixengelmann/ACTRin-R/tree/ee01519

Engelmann, F., Jäger, L. A., \& Vasishth, S. (2019). The effect of prominence and cue association on retrieval processes: A computational account. Cognitive Science, 43(12), e12800.

Engelmann, F., Vasishth, S., Engbert, R., \& Kliegl, R. (2013). A framework for modeling the interaction of syntactic processing and eye movement control. Topics in Cognitive Science, 5(3), 452-474. https://doi.org/10.1111/tops.12026

Findlay, J. M., \& Walker, R. (1999). A model of saccade generation based on parallel processing and competitive inhibition. Behavioral and Brain Sciences, 22(4), 661-674. https://doi.org/ $10.1017 / \mathrm{s} 0140525 \times 99002150$

Frazier, L., \& Rayner, K. (1982). Making and correcting errors during sentence comprehension: Eye movements in the analysis of structurally ambiguous sentences. Cognitive Psychology, 14(2), 178-210. 
Gordon, P. C., Hendrick, R., Johnson, M., \& Lee, Y. (2006). Similarity-based interference during language comprehension: Evidence from eye tracking during reading. Journal of Experimental Psychology: Learning, Memory, and Cognition, 32(6), 1304-1321.

Just, M. A., \& Carpenter, P. A. (1980). A theory of reading: From eye fixations to comprehension. Psychological Review, 87(4), 329-354.

Kliegl, R., Grabner, E., Rolfs, M., \& Engbert, R. (2004). Length, frequency, and predictability effects of words on eye movements in reading. European Journal of Cognitive Psychology, 16(1-2), 262-284. https://doi.org/10.1080/09541440340000213

Laloy, E., \& Vrugt, J. A. (2012). High-dimensional posterior exploration of hydrologic models using multiple-try DREAM(ZS) and high-performance computing. Water Resources Research, 48(1). https://doi.org/10.1029/2011wr010608

Lewis, R. L., \& Vasishth, S. (2005). An activation-based model of sentence processing as skilled memory retrieval. Cognitive Science, 29(3), 375-419. https : / / doi . org / 10 . 1207 / s15516709cog0000_25

McConkie, G., Kerr, P., Reddix, M., \& Zola, D. (1988). Eye movement control during reading: I. The location of initial eye fixations on words. Vision Research, 28(10), 1107-1118. https: //doi.org/10.1016/0042-6989(88)90137-X

Mertzen, D., Laurinavichyute, A., Dillon, B., Engbert, R., \& Vasishth, S. (2020). A cross-linguistic investigation of proactive, similarity-based retrieval interference in sentence comprehension: No support from English, German and Russian eye-tracking data. https://doi.org/10. 31234/osf.io/t2j8v

Rabe, M. M., Chandra, J., Krügel, A., Seelig, S. A., Vasishth, S., \& Engbert, R. (in press). A Bayesian approach to dynamical modeling of eye-movement control in reading of normal, mirrored, and scrambled texts. Psychological Review. https://doi.org/10.1037/rev0000268

Rayner, K. (1998). Eye movements in reading and information processing: 20 years of research. Psychological Bulletin, 124(3), 372-422. https://doi.org/10.1037/0033-2909.124.3.372

Reich, S., \& Cotter, C. (2015). Probabilistic forecasting and bayesian data assimilation. Cambridge University Press. https://doi.org/10.1017/CBO9781107706804

Reichle, E. D., Pollatsek, A., Fisher, D. L., \& Rayner, K. (1998). Toward a model of eye movement control in reading. Psychological Review, 105(1), 125-157.

Salvucci, D. D. (2001). An integrated model of eye movements and visual encoding. Cognitive Systems Research, 1(4), 201-220.

Schütt, H. H., Rothkegel, L. O., Trukenbrod, H. A., Reich, S., Wichmann, F. A., \& Engbert, R. (2017). Likelihood-based parameter estimation and comparison of dynamical cognitive models. Psychological Review, 124(4), 505-524. https://doi.org/10.1037/rev0000068

Seelig, S. A., Rabe, M. M., Malem-Shinitski, N., Risse, S., Reich, S., \& Engbert, R. (2020). Bayesian parameter estimation for the SWIFT model of eye-movement control during reading. Journal of Mathematical Psychology, 95, 102313. https://doi.org/10.1016/j.jmp.2019. 102313

Snell, J., van Leipsig, S., Grainger, J., \& Meeter, M. (2018). OB1-Reader: A model of word recognition and eye movements in text reading. Psychological Review, 125(6), 969-984. https: //doi.org/10.1037/rev0000119

Tabor, W., Galantucci, B., \& Richardson, D. (2004). Effects of merely local syntactic coherence on sentence processing. Journal of Memory and Language, 50(4), 355-370. 
ter Braak, C. J. F., \& Vrugt, J. A. (2008). Differential evolution Markov chain with snooker updater and fewer chains. Statistics and Computing, 18(4), 435-446. https://doi org/10.1007/ s11222-008-9104-9

Van Dyke, J. A., \& McElree, B. (2006). Retrieval interference in sentence comprehension. Journal of Memory and Language, 55(2), 157-166. https://doi.org/10.1016/j.jml.2006.03.007

Vasishth, S., \& Engelmann, F. (2021). Sentence comprehension as a cognitive process: A computational approach [Under contract with Cambridge University Press]. https://vasishth.github. io/RetrievalModels/

Veldre, A., Yu, L., Andrews, S., \& Reichle, E. D. (2020). Towards a complete model of reading: Simulating lexical decision, word naming, and sentence reading with über-reader. Proceedings of the 42nd Annual Conference of the Cognitive Science Society. https://hdl.handle. net/2123/22990

Vrugt, J. A., ter Braak, C., Diks, C., Robinson, B. A., Hyman, J. M., \& Higdon, D. (2009). Accelerating Markov chain Monte Carlo simulation by differential evolution with self-adaptive randomized subspace sampling. International Journal of Nonlinear Sciences and Numerical Simulation, 10(3). https://doi.org/10.1515/ijnsns.2009.10.3.273 